\title{
Fuzzy constraints in the Truck and Trailer Routing Problem
}

\author{
Isis Torres ${ }^{1}$, Alejandro Rosete ${ }^{1}$, Carlos Cruz ${ }^{2}$, José Luis Verdegay ${ }^{2}$ \\ ${ }^{1}$ Instituto Superior Politécnico José Antonio Echeverría, La Habana, Cuba \\ ${ }^{2}$ Universidad de Granada, Granada, España \\ \{itorres, rosete\}@ceis.cujae.edu.cu, \{carloscruz, verdegay\}@decsai.ugr.es
}

\begin{abstract}
Techniques based on Soft Computing are useful to solve real-world problems where decision makers use subjective knowledge when making decisions. In many problems in transport and logistics it is necessary to take into account that the available knowledge about the problem is imprecise or uncertain. Truck and Trailer Routing Problem (TTRP) is one of most recent and interesting problems in transport routing planning. Most of models used in the literature assume that the data available are accurate; for this reason it would be appropriate to focus research toward defining TTRP models for incorporating the uncertainty present in their data.
\end{abstract}

Keywords: Decision making, fuzzy logic, Soft Computing, TTRP

\section{Truck and Trailer Routing Problem}

Intelligent transport systems are advanced applications which aim to provide innovative services relating to different modes of transport and traffic management and enable various users to be better informed and make safer, more coordinated, and "smarter" use of transport networks. Vehicle Routing Problem (VRP) is a generic name given to a whole class of problems where it is needed to design a set of routes. In the VRP a fleet of vehicles has to be routed from one or several depots to serve a set of geographically dispersed cities or customers, subject to side constraints. The goal is to design vehicle routes originating from and terminating at the central depot to fulfill each customer's demand so that the total cost is minimized. The total demand on each route should not exceed the vehicle capacity and each customer can only be serviced once by exactly one vehicle [1][2][3][4][5]. There exists a wide variety of VRPs [6][7][8][9][10]; among them is distinguished the Truck and Trailer Routing Problem (TTRP).

The TTRP is a combinatorial optimization problem and an extension of the well-known VRP. The main difference between TTRP and VRP consist of the use of trailers; a commonly neglected feature in the VRP.
In the standard TTRP, the customers are geographically dispersed and they have an associated demand. The customers are serviced by a fleet of vehicles (truck pulling a trailer) with known capacity. However, due to practical constraints, including government regulations, limited maneuvering space at customer site, road conditions, etc., some customers may only be serviced by a truck.

The fleet of vehicles in a TTRP consists of trucks and trailers. Once a trailer is assigned to a truck; it may not be assigned to another truck. A truck with a trailer is a complete vehicle, while that a truck without a trailer is a pure truck. All trucks and all trailers have identical capacities. However it can be considered as a heterogeneous fleet capacity due to vehicle types that can be used: complete vehicle and pure truck. The TTRP considers two forms of customer types: a customer who is accessible with or without a trailer (vehicle customer), and one who is only accessible without a trailer (truck customer).

All these characteristics generate different types of routes in a TTRP solution as illustrated in Figure 1. These routes are originating from and terminating at a central depot and they are limited by capacity of vehicle used.

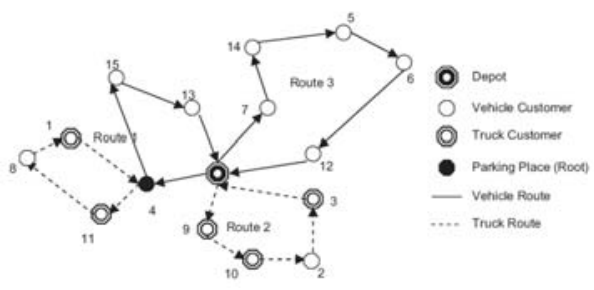

Fig. 1: Routes types in the TTRP (Taken from [15]).

There are three types of routes in a TTRP solution. The types of routes are:

- Complete Vehicle Route (CVR): Consisting of a main tour traveled by a complete vehicle, and at least one sub-tour traveled by the truck alone.

- Pure Truck Route (PTR): This type of route is traveled by a truck alone and are visited both customers. 
- Pure Vehicle Route (PVR): It is the tour traveled by a complete vehicle and contains only vehicle customers without any sub-tour.

The growing interest in TTRP is motivated by its practical relevance in many real world problems. Also, the broad range of actual applications have caused the definition of many TTRP variants with real-world constraints.

\subsubsection{Related problems}

The term "Truck and Trailer Routing Problem" was first used in 2002 by I-Ming Chao [11]. However, previous works were developed to solve practical situation present in real-life with similar characteristics to TTRP. These problems are variants of VRP and can be viewed as closer approaches to TTRP.

The first work was presented by Semet and Taillard in [12]. The problem occurred at a regional level of one of the major chain store in Switzerland. The company owns a heterogeneous fleet of vehicles consisting of 21 truck and 7 trailers. This Swiss chain store involves 45 grocery stores located in areas that did not allow access with trailer. The goal is to determine a transportation schedule using a heterogeneous fleet that minimizes the transportation costs. The authors studied a real-life VRP that deals with new features such as, the heterogeneous character of the fleet, in the sense that utilization costs are vehicle-dependent, time windows and the use of trailers under accessibility restrictions. Also, the customer's trailer-store cannot be serviced in a sub-tour. That paper proposed a heuristic method based on clustering methods and a standard Tabu search method for finding good solutions.

Two years later is modeled the Partial Accessibility Constrained Vehicle Routing Problem (PACVRP) [13]. PACVRP is an extension of the VRP. It takes into account the partial accessibility constraint introduced in previous works [12]. In this case the partial accessibility constraint consists of forbid same customers to be served by a truck and its trailer. Although this problem is very similar to TTRP, there are differences between them such as:

- All available trucks are used.

- The depot can be visited only once in a route.

- The number of trailers must be determined.

- The number of sub-tours assigned to a parking place is restricted to a maximum of one.

The author provided an integer programming formulation and proposed a two-phase heuristic method. Also, to solve a combinatorial problem that has some similarities with the Generalized Assignment Problem (GAP) he proposes an enumerative procedure using Lagrangian relaxation.
Another work is the study by Gerdessen in 1996 on Vehicle Routing Problems with Trailers (VRPT) [14]. In this problem the combination of truck and trailer is called vehicle or complete vehicle. However, the use of a complete vehicle may cause problem when serving customer with constraints. Time and trouble could be saved if these customers were served by the truck only. Also, an additional advantage will be the saving of fuel and drives faster. The VRPT is very similar to the classic TTRP, but the author simplified the model with a set of assumptions. The VRPT differs from TTRP mainly in:

- All customers have unit demand.

- Each trailer is parked exactly once.

- Each customer site can be used as a parking place.

- Each customer is assigned a maneuvering cost instead of customer types.

Summarizing, before the formal definition of TTRP there were real-life applications with similar characteristics to this kind of problem. Although, all they present differences with respect to the classic TTRP.

\subsubsection{Formal Definition}

The TTRP can be formally defined on an undirected graph $G=(V, A)$, where $V=\left\{v_{0}, v_{1}, \ldots, v_{n}\right\}$ is a vertex set and $A=\left\{\left(v_{i}, v_{j}\right): v_{i}, v_{j} \in V, i \neq j\right\}$ is the set of edges. The vertices represent customer, except the vertex $v_{0}$ that be corresponds to central depot. There is a vector of the customer demands $d_{i}$ and each customer $i \in V \backslash v_{0}$ has a non-negative demand $d_{i}>0$.

The access constraints create a partition of $V$ into two subsets: the subset of truck customer $V_{c} \subseteq V$ accessible only by truck and the subset of vehicle customer $V_{v} \subseteq V$ accessible either by truck or by truck with trailer.

$C$ is a matrix of non-negative cost. Each edge $(i, j)$ $\in V$ is associated with a cost $c_{i j}$ that represents the travel time required on the edge or the travel distance between vertex $v_{i}$ and vertex $v_{j}$. A heterogeneous fleet consist of $m_{c}$ trucks and $m_{r}$ trailers, where $m_{r} \leq m_{c}$. The capacities of the trucks and the trailers are $q_{c}$ and $q_{r}$ respectively. Each vehicle $k$ is assigned to a route $R_{k}$.

A route in the TTRP is composed of a partition of $V: R_{1}, \ldots, R_{k}$ and a permutation $\delta_{k}$ of $R_{k}$ specifying the order of the customers on route. Each route is originating from and terminating at a central depot: $R_{k}=\left\{v_{0}, v_{1}, \ldots, v_{n+1}\right\}$, where $v_{0}=v_{n+1}$ denotes the depot. Each route is classified as route of complete vehicle $\left(R_{c v}\right)$ or route of pure truck $\left(R_{p t}\right)$.

Thus, the goal of the TTRP is to find a set of least cost vehicle routes that start and end at the central depot such that each customer is serviced exactly once and the total demand of any vehicle route does not 
exceed the total capacity of the allocated vehicles used in that route; and the number of required trucks and trailers is not greater than $m_{c}$ and $m_{r}$, respectively.

\subsubsection{Variants and Extensions}

Few research efforts have been devoted to study the TTRP. However, there are some papers on several TTRP variants. Some important variants of TTRP are Truck and Trailer Routing Problem with Time Windows (TTRPTW) and Relaxed Truck and Trailer Routing Problem (RTTRP). Also, there are others such as Multi-Depot Truck and Trailer Routing Problem (MDTTRP), Single Truck and Trailer Routing Problem with Satellite Depots (STTRPSD), Periodic Truck and Trailer Routing Problem (PTTRP), etc. The following section describes some of these variants:

Relaxed Truck and Trailer Routing Problem (RT$T R P)$ : This variant relaxes the fleet size constraint for the TTRP [16]. In the TTRP there are not fix costs associated with the vehicles although there are limitations on the number of available trucks and available trailers. Thus, it is possible to construct better vehicle routes by utilizing more vehicles or allowing vehicles to take on multiple trips. Further, if the reduction in costs resulting from such relaxation is significant, it may be worthwhile to acquire or lease extra vehicles provided that the acquisition or lease costs can be justified. The resulting RTTRP can be also used to determine a better fleet mix. Therefore, it is reasonable to relax the fleet size constraint with the goal to further reduce the total routing cost.

Truck and Trailer Routing Problem with Time Windows (TTRPTW): In many real-world routing applications the time windows constraints are present. The TTRPTW can be regarded as a variant of the Vehicle Routing Problem with Time Windows (VRPTW). This model is an variant of the TTRP [17]. The problem definition is similar, it only add in each vertex $v_{i}$ a time window $\left(e t_{i}, l t_{i}\right)$ and a service time $s t_{i}$. The parameter $e t_{i}$ and $l t_{i}$ denote the earliest time and latest time that the service to customer $i$ can start, respectively and $s t_{i}$ denotes the time required to service customer $i$. In this problem must be fulfilled that each customer is serviced within their specific time windows

Single Truck and Trailer Routing Problem with Satellite Depots (STTRPSD): The STTRPSD is a generalization of the VRP. In the STTRPSD a vehicle composed of truck with a detachable trailer serves the demand of a set of customers reachable only by the truck without the trailer. There is a set of parking locations called trailer points or satellite depots, where it is possible to detach the trailer and to transfer products between the truck and the trailer. Each customer is assigned to one trailer point in a feasible solution of the STTRPSD. Consequently, trailer points with assigned customers are said to be open [18][19].

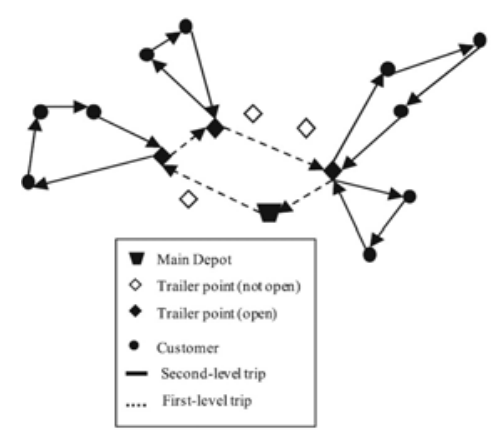

Fig. 2: STTRPSD solution (Taken from [18]).

The tour is traveled in two levels (see Figure 2). The first level trip departing from the main depot is performed by the truck with the trailer and visits the subset of open trailer points. The second level trip serves the demand of those customers reachable only by the truck without the trailer. The second level trip starts and ends at the allocated trailer point and the total load should not exceed the truck capacity. The goal of the STTRPSD is to minimize the total length of the trips.

Furthermore, several authors have defined TTRP extensions such as:

Generalized Truck and Trailer Routing Problem (GTTRP): In [20] the author presented GTTRP which is a rather complex generalization motivated by a realworld scenario. Here trucks and trailers can be either collection vehicles or support vehicles. Collection vehicles are used to collect the supplies of customers, while support vehicles are used as "mobile depots" that cannot visit customers. A trailer may be pulled by different trucks during the course of its tour, and the load of the truck may be transferred from any vehicle to any other vehicle during a tour. Also, any intermediate locations en-route can be used either for parking or for load transfer.

Extended Truck and Trailer Routing Problem (ETTRP): ETTRP is an extended version of the TTRP, in which the main objective is to minimize the total length of all constructed routes. This thesis expands on the model initially defined by Chao by introducing several additional constraints, which mimic problems that could arise in a real-world application. Thus, both time window constraints as well as load constraints are considered [21].

\subsubsection{Strategies applied to TTRP}

Since TTRP itself is a very difficult combinatorial optimization problem it is usually tackled by approximate algorithms because no exact algorithm can be guaranteed to find optimal tours within reasonable comput- 
ing time when the number of customers is large. This is due to the NP-Hardness of the problem [22].

The only exact approach for the TTRP is due to Drexl [23]. The author developed a branch and price algorithm for the TTRP, where the algorithm had only been tested on relatively small instances of the TTRP. Otherwise, approximate algorithms are very popular to solve this type of problem. There exists a wide variety of metaheuristics proposed to solve TTRP. Each one have achieved satisfactory results for routing problems, between them are:

Both Chao [11] and Scheuerer [24] solved the TTRP by a 2-phase approach. In the first phase, construction heuristics were used to obtain an initial TTRP solution. The initial solution was then improved by a Tabu Search (TS) algorithm in the second phase.

Lin et al. in [15] developed a very effective Simulated Annealing (SA) based heuristic to the TTRP and obtained results that are competitive with those obtained by Scheuerer. Also, it applied a route combination procedure to reduce the number of required trucks and trailers.

In [25] the authors designed a mathematical programming based heuristic that also employs the cluster-first route-second approach. Their method solves two subproblems sequentially. The first, called customer-route assignment problem (CAP) and the second, the route definition problem (RDP). The authors embedded these two models within an iterative mechanism that adds new constraints to the CAP based on the information of the RDP solution. This restarting mechanism is intended to diversify the search, and includes a Tabu search mechanism that forbids (in the CAP) customers route assignments already explored in previous iterations of the algorithm.

Villegas et al. proposed in [26] solved the TTRP using a route-first, cluster-second procedure embedded within a hybrid metaheuristic based on a Greedy Randomized Adaptive Search Procedures (GRASP), Variable Neighborhood Search (VNS), and Path Relinking. Likewise, the same authors solved the STTRPSD with a multi start evolutionary local search and a hybrid GRASP/VND [18]. These metaheuristics use a routefirst cluster-second procedure and a VND as building blocks.

Lastly, in [27] it is applied a heuristic approach to the TTRP which combines local search and large neighborhood search as well as standard metaheuristics control strategies. According to [28] this approach can be applied to several variants of VRPs. The author has developed a VRP-software framework based on this heuristic concept and he shown how solvers for different VRPs in a rather simple and flexible manner.

\section{A Soft Computing - based approach}

Different sources of uncertainty in transport problems are present in real world contexts, and only approximate, vague and imprecise values are known. Therefore, often the decision maker cannot formulate all the data precisely. In general, users and decision makers diligently establish measurements based on observations and perceptions which determine the problem parameters and in the same way affect the evaluation of objectives and obtained solutions.

The TTRP is a problem which by its nature favors the presence of vagueness, imprecision and uncertainty in the information handled. Nevertheless, the models used in the literature assume that the data available are accurate; consideration does not correspond with reality. For this reason it would be appropriate to focus research toward defining TTRP models for incorporating the uncertainty present in the data.

The first case of optimization problems with fuzzy approach appeared in the literature more four decades ago [29], in an article which put forward the now classical key concepts of constraint, objective and fuzzy optimal decision. Later, in 1974 Tanaka et al. in [30] and Zimmermann in [31], are the pioneers on Fuzzy Linear Programming (FLP) based on the concept of Bellman and Zadeh, for decision making under fuzzy conditions.

Fuzzy optimization models and methods has been one of the most and well studied topics inside broad area SC. Particularly relevant is the field of FLP that constitutes the basis for solving Fuzzy Optimization Problems. FLP and related problems have been extensively analyzed and many papers have been published displaying a variety of formulations and approaches, such as transportation, production planning, water supply planning and resource management, forest management, bank management, portfolio selection, pattern classification, and others [32][33].

The classic problem of LP is to find the maximum or minimum values of a linear function subject to constraints that are represented by linear equations or inequalities. The most general formulation of the LP problem is:

$$
\begin{gathered}
\operatorname{Max} z=c x \\
A x \leq b \\
x \geq 0
\end{gathered}
$$

The vector $x=\left(x_{1}, x_{2}, \ldots, x_{n}\right) \in \mathbb{R}^{n}$ represents the decision variables. The objective function is denoted by $z$, the numbers $c_{j}$ are coefficients and the vector $c=\left(c_{1}, c_{2}, \ldots, c_{n}\right) \in \mathbb{R}^{n}$ is known as the cost vector. The matrix $A=\left[a_{i j}\right] \in \mathbb{R}^{n \times m}$ is called the constraint or technological matrix and the vector 
$b=\left(b_{1}, b_{2}, \ldots, b_{m}\right) \in \mathbb{R}^{m}$ represents the independent terms or right-hand-side of the constraints.

The formulation of a linear programming problem under fuzzy environment depends on what and where the fuzziness is introduced. In the last past years several kinds of FLP problems have appeared in the literature [34], but the three main are:

- Linear programming problems with a fuzzy objective, i.e. with fuzzy numbers defining the costs of the objective function.

- Linear programming problems with a fuzzy constraint set, i.e. with a feasible set defined by fuzzy constraints.

- Linear programming problems with fuzzy numbers defining the coefficients of the technological matrix.

In this work, we will focus on a general parametric idea in order to transform fuzzy problems into many classical problems [35][36][37]. This fuzzy approach to dealing with a type of imprecision associated with vague and imprecise nature of linguistic terms used in the problem, in addition to tolerance in the evaluation of objectives and constraints.

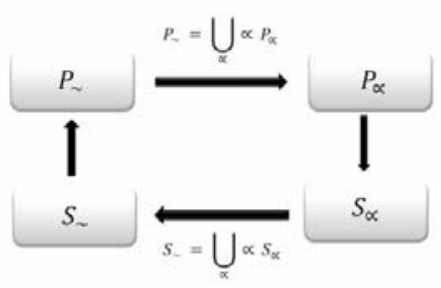

Fig. 3: Parametric idea.

This parametric approach is divided into two parts (see Figure 3): transforming a fuzzy problem into a classical parametric problem with a parameter $\alpha$ representing the makerï $\_\frac{1}{2}$ s satisfaction level (which belongs to the interval $[0,1]$ ); a mathematical formulation of the classical parametric problem that is equivalent to the original fuzzy problem. It is solved each different $\alpha$ values using conventional linear programming techniques. Since 1990, Delgado et al. [38] showed that the set of solutions achieved by this approach contains the solutions reached by other different approaches [30][31], which solve fuzzy linear programming problems too.

An idea of application of this approach is the case in which a decision maker assumes that he can tolerate violations in the accomplishment of the constraints; i.e. he permits the constraints to be satisfied "as well as possible". The associated problem is represented as follows

$$
\begin{gathered}
\operatorname{Max} z=c x \\
A x \leq_{f} b \\
x \geq 0
\end{gathered}
$$

where the symbol $\leq_{f}$ indicates the imprecision of the constraints and where each fuzzy constraint $a_{i} x \leq_{f}$ $b_{i}$ is modeled by means of a membership function $\mu_{i}$ : $\mathbb{R} \rightarrow[0,1]:$

$$
\mu_{i}(x)= \begin{cases}1 & \text { if } a_{i} x \leq b_{i} \\ f_{i}\left(a_{i} x\right) & \text { if } b_{i} \leq a_{i} \leq b_{i}+\tau_{i} \\ 0 & \text { if } b_{i}+\tau_{i} \leq a_{i} x\end{cases}
$$

These functions express that the decision maker is tolerating violations in each constraint up to a value of $b_{i}+\tau_{i}(\tau$ is referred to as a violation tolerance level). Functions $f_{i}$ are assumed to be non decreasing and continuous for these constraints. Function $\mu_{i}$ is defined for each $x$ and it gives for $x$ its accomplishment degree on the $i$-th constraint. Graphically we obtain a picture as shown in Figure 4.

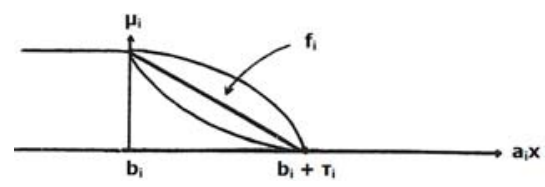

Fig. 4: Examples of fuzzy membership functions (Taken from [36]).

Lastly, making use of the Decomposition Theorem for fuzzy sets to represent a fuzzy set by means of its $\alpha$-cuts, and to work, then, on these classical sets instead of the fuzzy ones. The problem (2) can be easily transformed into a parametric programming problem as follows:

$$
\begin{gathered}
\text { Max } z=c x \\
A x \leq b+\tau(1-\alpha) \\
x \geq 0, \quad \alpha \in[0,1]
\end{gathered}
$$

where $\tau\left(\tau_{1}, \tau_{2}, \ldots, \tau_{m}\right) \in \mathbb{R}^{m}$ is the tolerance level vector.

The alternative presented proposes the application of the fuzzy logic is a way to describe this vagueness mathematically. Thus, one could obtain models that tolerate the uncertainty and tries to satisfy before to optimize. This proposal has been exploited in VRP variants [39][40][41][42]; however the complexity of TTRP, as well as for the recent of topic has not been applied in this problem.

For example, in the case of TTRP the constraints associated to the capacity of the vehicles can be represented by: 


$$
\begin{aligned}
\sum d_{i} x_{i}^{k} \leq q_{c} \quad k & =1, \ldots, m_{c}{ }^{\prime} \\
\sum d_{i} x_{i}^{k} \leq q_{c}+q_{r} \quad k & =1, \ldots, m_{r}
\end{aligned}
$$

where $m_{c}{ }^{\prime}$ is the number of trucks available after serving the routes of complete vehicle $\left(R_{c v}\right)$. Our model considers constraints as fuzzy and admits the violation of these constraints according with a value of tolerance $\tau$.

$$
\begin{gathered}
\sum d_{i} x_{i}^{k} \leq_{f} q_{c} \Longleftrightarrow \\
\sum d_{i} x_{i}^{k} \leq q_{c}+\tau_{i}(1-\alpha) \\
\sum d_{i} x_{i}^{k} \leq_{f} q_{c}+q_{r} \Longleftrightarrow \\
\sum d_{i} x_{i}^{k} \leq\left(q_{c}+q_{r}\right)+\tau_{i}(1-\alpha)
\end{gathered}
$$

The results obtained for each $\alpha$ value generate a set of solutions and then the Representation Theorem can be used to integrate all these specific $\alpha$ solutions. This fuzzy solution is found from the solution of the parametric LP problem.

\subsection{Computational results}

In order to illustrate the above described approach, we are going to focus on a problem with fuzzy constraints. The proposal was coded in Java and compiled using Eclipse 4.2.1. It was then applied to one TTRP benchmark instances created by Chao [11] (see Table 1) on a Core Duo $1.80 \mathrm{GHz}$ PC with 4GB RAM under Microsoft Windows 7 operating system.

\begin{tabular}{|c|c|}
\hline \multicolumn{2}{|c|}{ Problem \# 1 } \\
\hline Features & Values \\
\hline Number of Customers & 50 \\
Vehicle Customers & 38 \\
Truck Customers & 12 \\
Total Request & 777 \\
Number Trucks & 5 \\
Capacity Truck & 100 \\
Number Trailer & 3 \\
Capacity Trailer & 100 \\
\hline
\end{tabular}

Table 1: Dimension of problem 1. Reported by [11].

In the initial experiments, the following combinations of parameters were tested:

- Algorithm = HillClimbing

- Executions $=30$

- Iterations $=100000$

- Penalty $=125$

- $\tau=10$

- $\alpha=\{0,0.1,0.2,0.3,0.4,0.5,0.6,0.7,0.8,0.9$, $1.0\}$
The results obtained are presented in Table 2 and Table 3. The best value of the objective function (Min) and the average behavior (Avg) of the solutions obtained is shown for each value of $\alpha$. Values contained in brackets are associated with the routes number of each type $\left(R_{v c}, R_{c p}\right)$ obtained in the solution. Results in Table 3 were obtained applying a strategy of penalty; which involves to add a penalty cost to the objective function for each extra vehicle requiring the solution.

\begin{tabular}{|c|c|c|}
\hline \multicolumn{3}{|c|}{$\tau=10$} \\
\hline$\alpha$ & Min & Avg \\
\hline 0.0 & $631.05_{(4,2)}$ & 714.37 \\
0.1 & $613.36_{(3,3)}$ & 719.26 \\
0.2 & $630.42_{(4,1)}$ & 720.29 \\
0.3 & $654.19_{(3,2)}$ & 712.32 \\
0.4 & $627.06_{(4,2)}$ & 706.99 \\
0.5 & $643.98_{(2,4)}$ & 725.69 \\
0.6 & $619.82_{(3,3)}$ & 714.38 \\
0.7 & $642.66_{(3,3)}$ & 721.21 \\
0.8 & $617.83_{(4,1)}$ & 706.28 \\
0.9 & $631.23_{(3,3)}$ & 737.98 \\
1.0 & $616.77_{(4,2)}$ & 729.38 \\
\hline
\end{tabular}

Table 2: Results obtained by the fuzzy approach.

Also, results show that the trend is to obtain better solutions when the level of tolerance increases in the constraint. Similarly, the results worsen for higher $\alpha-$ cuts values, in other words, when the compliance is less flexible constraints. It is noteworthy that most of the results in Table 2 correspond to infeasible solutions, in a different way to the results in Table 3 . In this case the best solutions are feasible and all of the available vehicles are used.

\begin{tabular}{|c|c|c|}
\hline \multicolumn{3}{|c|}{$\tau=10$} \\
\hline$\alpha$ & Min & Avg \\
\hline 0.0 & $612.06_{(3,2)}$ & 748.48 \\
0.1 & $650.89_{(3,2)}$ & 750.33 \\
0.2 & $661.43_{(3,2)}$ & 748.86 \\
0.3 & $672.91_{(3,2)}$ & 756.03 \\
0.4 & $641.61_{(3,2)}$ & 762.38 \\
0.5 & $623.59_{(3,2)}$ & 770.55 \\
0.6 & $648.37_{(3,2)}$ & 783.61 \\
0.7 & $678.23_{(3,2)}$ & 791.91 \\
0.8 & $675.87_{(3,2)}$ & 789.76 \\
0.9 & $625.57_{(3,2)}$ & 803.69 \\
1.0 & $706.85_{(3,2)}$ & 824.72 \\
\hline
\end{tabular}

Table 3: Results obtained by the fuzzy approach with penalty strategy.

The most significant aspect of the parametric method is that it allows obtaining a set of fuzzy solutions to the original problem. Thus, the decision 
maker has a set of solutions, not just a single solution. This will allow the decision maker to select the most appropriate solution according to their actual needs, to the knowledge of the information available, and their way of making decision.

\section{Conclusion}

Truck and Trailer Routing Problem, a variant of the Vehicle Routing Problem in which a subset of the vehicles is allowed to pull trailers because of the benefit of increased capacity. It is one of the most interesting problems at this time in transport routing planning. Unfortunately to date, not much research has been done about this problem. Some of their most known variants and solutions applied to solve it were described here. All these models assume that the data available are accurate. This consideration does not correspond with reality where the vagueness appears in a natural way. For this reason it would be appropriate to focus research toward defining TTRP models for incorporating the uncertainty present in the data, and hence it makes perfect sense to think of Soft Computing techniques. This paper is only the beginning of future works about of how to solve the TTRP applying these techniques. Thus, a known fuzzy parametric approach is proposed as a general method to solve this problem when its elements are vague, imprecise and inexact. The authors aim to extend the line of research involving Fuzzy Programming problems in order to try to solve practical real-life problems by facilitating the building of Decision Support Systems.

\section{Acknowledgements}

This paper has been partially supported by the following projects: TIN2011-27696-C02-01 and P11-TIC8001. Also, the authors wish to thank to AUIP.

\section{References}

[1] G. Dantzig and J. Ramser, The truck dispatching problem, Management Science, 6:80-91,1959.

[2] B. Gillett and L. Miller, A heuristic algorithm for the vehicle dispatch problem. Operations Research, 22:340-349, 1974.

[3] L. Bodin, B. Golden, A. Assad and M. Ball, Routing and Scheduling of Vehicles and Crews: The State of The Art. Computers and Operations Research, 10:62-212, 1983.

[4] B. Golden and A. Assad. Vehicle Routing: Methods and Studies, North-Holland Studies in Telecommunication, Amsterdam, 1988.

[5] P. Toth and D. Vigo. The Vehicle Routing Problem, Monographs on Discrete Mathematics and Applications Series, Society for Industrial and Applied Mathematics (SIAM), Philadelphia, 2002.
[6] M. Goetschalckx, C. Jacobs-Blecha, The Vehicle Routing Problem with Backhauls: An Optimization Based Approach. Proceedings of the $2^{\text {nd }}$ Industrial Engineering Research Conference, pages 504509, May 26-27, Los Angeles (California), 1993.

[7] M. Dror, G. Laporte and P. Trudeau, Vehicle routing with split deliveries, Discrete Appl. Math., 50:239-254, 1994.

[8] M. Solomon, Algorithms for the Vehicle Routing Problem with Time Windows, Transportation Science, 29:156-166, 1995.

[9] S. Baptista, R. Oliveira and E. Zúquete, A Period Vehicle Routing Case Study, European Journal of Operational Research, 139:220-229, Elsevier, 2002.

[10] T. Ralphs, L. Kopman, W. Pulleyblank and L. Trotter Jr, On the Capacitated Vehicle Routing Problem, Math. Program., 94:343-359, SpringerVerlag, 2003.

[11] I-M. Chao, A tabu search method for the truck and trailer routing problem, Computers and Operation Research, Elsevier, 29:33-51, 2002.

[12] F. Semet and E. Taillard, Solving real-life vehicle routing problems efficiently using tabu search, $A n$ nals of Operations Research, Elsevier, 41:469-488, 1993.

[13] F. Semet, A two-phase algorithm for the partial accessibility constrained vehicle routing problem, Annals of Operations Research, Elsevier, 61:45-65, 1995.

[14] J. Gerdessen, Vehicle routing problem with trailer problem, European Journal of Operational Research, Elsevier, 93:135-147, 1996.

[15] S-W. Lin, V.F. Yu and S-Y. Chou, Solving the truck and trailer routing problem based on a simulated annealing heuristic, Computers and Operation Research, 36:1683-1692, Elsevier, 2009.

[16] S-W. Lin, V.F. Yu and S-Y. Chou, A note on the truck and trailer routing problem, Expert Systems with Application, 37:899-903, Elsevier, 2010.

[17] S-W. Lin, V.F. Yu and Ch-Ch. Lu, A simulated annealing heuristic for the truck and trailer routing problem with time windows, Expert Systems with Application, 38:15244-15252, Elsevier, 2011.

[18] J.G. Villegas, C. Prins, C. Prodhon, A.L. Medaglia and N. Velasco, GRASP/VND and multi-start evolutionary local search for the single truck and trailer routing problem with satellite depots, Engineering Applications of Artificial Intelligence, 23:780-794, Elsevier, 2010.

[19] J.G. Villegas, C. Prins, C. Prodhon, A.L. Medaglia and N. Velasco, GRASP/Evolutionary Local Search Hybrids for a Truck and Trailer Routing Problem In M. Verleysen, editor, proceedings of The VIII Metaheuristics International Conference (MIC 2009), d-side pub., pages 211-216, July 1316, Hamburg (Germany), 2009. 
[20] M.A. Drexl, Branch and price and heuristic colum generation for the generalized truck and trailer routing problem, Revista de Métodos Cuantitativos para la Economía y la Empresa, 12:5-38, 2011.

[21] R. Zitz. Algorithms for the Truck and Trailer Routing Problem, Department of Mathematics and Computer Science, University of Southern Denmark, Syddansk Universitet Institut for Matematik og Datalogi, 2010.

[22] M.R. Garey and D.S. Johnson. Computers and intractability: a guide to the theory of NPcompleteness, W. H. Freeman \& Co, New York 1979.

[23] M.A. Drexl, A branch and price algorithm for the truck and trailer routing problem. Technical Report, N. 27/2006 Deutsche Post Endowed Chair of Optimization of Distribution Networks, RWTH Aachen University, Germany, October 2007.

[24] S. Scheuerer, A tabu search heuristic for the truck and trailer routing problem, Computers and Operation Research, 33:894-909, Elsevier, 2006.

[25] M. Caramia and F. Guerriero, A heuristic approach for the truck and trailer routing problem, Journal of the Operational Research Society, 61:1168-1180, 2010.

[26] J.G. Villegas, C. Prins, C. Prodhon, A.L. Medaglia and N. Velasco, A GRASP with evolutionary path relinking for the truck and trailer routing problem, Computers and Operation Research, 38:1319-1334, Elsevier, 2011.

[27] U. Derigs, M. Pullmann and U. Vogel, Truck and trailer routing-Problems, heuristics and computational experience, Computers \& $O$ Operation Research, 40:536-546, Elsevier, 2013.

[28] U. Vogel, A flexible metaheuristic framework for solving rich vehicle routing problems, Wirtschaftsinformatik und Operations-Research, Shaker, Germany, 2011.

[29] R.E. Bellman and L.A. Zadeh, Decision-making in a fuzzy environment, Management Science, Application Series, 17: B141-B164, 1970.

[30] H. Tanaka, T. Okuda and K. Asai, On fuzzy mathematical programming, Journal Cybernet,3: 37-46, 1974.

[31] H. Zimmermann, Optimization in fuzzy environment In M. Verleysen, editor, proceedings of the Int. TIMS and $46^{\text {th }}$ ORSA Conferenc, pages 211216, April 28-30, San Juan (Puerto Rico), 1974.

[32] Y. Lai and C. Hwang, Fuzzy mathematical programming: methods and applications. In Lecture Notes in in Economics and Mathematical Systems vol 394, Springer, 1992.

[33] N. Sahinidis, Optimization under uncertainty: State of the art and opportunities, Computers and Chemical Engineering, 28:971-983, 2004.

[34] A. Baykasoglu and T. Gcken, A review and clas- sification of fuzzy mathematical programs. Journal of Intelligent and Fuzzy Systems, 19:205-229, 2008.

[35] J. L. Verdegay, Fuzzy mathematical programming, Fuzzy Information and Decision Processes, 231-237, 1982.

[36] M. Delgado, J. Verdegay and M. Vila, A General Model for Fuzzy Linear Programming Fuzzy Sets and Systems, 29:21-29, 1989.

[37] R. Silva, J. Verdegay and A. Yamakami, Twophase method to solve fuzzy quadratic programming problems. In In IEEE International Conference on Fuzzy Systems, pages 1-6, 2007.

[38] M. Delgado, J. Verdegay and M. Vila, Relating different approaches to solve linear programming problems with imprecise costs, Fuzzy Sets and Systems, 37:33-42, 1990.

[39] J. Brito, F. J. Martínez, J. A. Moreno and J. L. Verdegay, A GRASP-VNS Hybrid for the Fuzzy Vehicle Routing Problem with Time Windows. In R. Moreno-Díaz, F. Pichler and A. QuesadaArencibia, editors, proceedings of the Computer Aided Systems Theory - EUROCAST 2009, Lecture Notes in Computer Science 5717, pages 825832, Springer-Verlag Berlin Heidelberg, 2009.

[40] B. Melian and J. L. Verdegay, Using Fuzzy Numbers in Network Design Optimization Problems, IEEE TRANSACTIONS ON FUZZY SYSTEMS, 19:797-806, 2011.

[41] J. Brito, F. J. Martínez, J. A. Moreno and J. L. Verdegay, ACO-GRASP-VNS Metaheuristic for VRP with Fuzzy Windows Time Constraints. In R. Moreno-Díaz, F. Pichler and A. QuesadaArencibia, editors, proceedings of the Computer Aided Systems Theory - EUROCAST 2011, Part I Lecture Notes in Computer Science 6927, pages 440-447, Springer-Verlag Berlin Heidelberg, 2012.

[42] J. Brito, J. A. Moreno and J. L. Verdegay, Transport route planning models based on fuzzy approach Iranian Journal of Fuzzy Systems, 9:141158, 2012. 